\title{
Study of the Impact of Rectangular Current Pulses on the Ten Tusscher-Panfilov Model of Human Ventricular Myocyte
}

\section{Boris B. Gorbunov}

Department of Biomedical Systems, National Research University of Electronic Technology, Moscow, Russia

Correspondence to: Boris B. Gorbunov, boris.b.gorbunov@org.miet.ru

Keywords: Human Ventricular Myocyte Model, Transmembrane Potential, Action Potential, Refractoriness, BeatBox Simulation Environment; Mechanism of Defibrillation

Received: June 21, $2017 \quad$ Accepted: July 18, $2017 \quad$ Published: July 21, 2017

Copyright $\odot 2017$ by authors and Scientific Research Publishing Inc.

This work is licensed under the Creative Commons Attribution International License (CC BY 4.0).

http://creativecommons.org/licenses/by/4.0/

\section{(c) (i) Open Access}

\section{ABSTRACT}

The behavior of the 2006 ten Tusscher-Panfilov model of human ventricular myocytes under the impact of periodic excitation impulses was studied in the BeatBox simulation environment. The cardiomyocyte model has a limited susceptibility to an forced higher frequency excitation rhythm. A high-frequency excitation rhythm can be forced by gradually increasing the frequency of excitation impulses. The mechanism of defibrillation pulse impact consists of presumably prolonging the refractoriness of cardiomyocytes which undermines their susceptibility for a long time to a forced high-frequency rhythm of fibrillation, as a result for which they hinder the propagation of a fibrillation wave. This is the only mechanism of defibrillation that was identified during the simulation. The threshold energy of a depolarizing defibrillation pulse prolonging the refractoriness of the cardiomyocyte varies depending on a delay relative to the excitation impulse (the excitation cycle phase) in a wide range (the maximum value exceeds the minimum by several thousand times). The results show differences in the mechanisms of impact on a cardiomyocyte between an excitation impulse and a monophasic defibrillation pulse.

\section{INTRODUCTION}

A number of previous theoretical studies have studied the effect on the cardiomyocyte membrane model of a single excitation pulse [1-8]. However, a study of the impact of an electrical defibrillation pulse on cardiomyocytes should consider the fact that during fibrillation a cardiomyocyte is not in a resting state and is subject to the impact of an abnormally frequent parasitic excitation wave while myocardial cardiomyocytes are in different phases of the excitation cycle. To stop fibrillation, it is necessary to change the 
state of cardiomyocytes in such a way that they become an obstacle to the propagation of the wave across the myocardium. This study has attempted to explain how to achieve change of its state to prevent the propagation of a fibrillation wave by using the cardiomyocyte model.

\section{MATERIALS AND METHODS}

The study was conducted in the BeatBox simulation environment [9] using the ten Tusscher-Panfilov 2006 model of human ventricular myocytes [10]. The simulation environment was set up in the Fedora operating system which was installed in the Oracle VM VirtualBox virtualization system on a PC using the Windows 7 operating system. We presented above the rationale for the use of the energy ratio as a parameter indicative of pulse energy efficiency [8]. A rectangular pulse waveform was chosen for the study as it had been described in the BeatBox scripts in elementary terms. Depolarizing excitation impulses with amplitude of $80 \mathrm{~mA} / \mathrm{cm}^{2}$ and duration of $0.5 \mathrm{~ms}$ at a predetermined interval were applied to the cardiomyocyte model. These are the standard parameters of the excitation pulses used in the simulation. Such a pulse provides excitation of the cardiomyocyte, and at the same time its duration is small in comparison with the duration of the action potential. At the beginning of the experiment the model parameters were initiated using the values saved upon completing the previous experiment.

In the experiments on the model, the possibility provided by the simulation environment to visualize the change in the transmembrane potential during a given time interval was used. The parameters of the acting pulses changed in the script, after which the appearance of the action potential or prolonging of the refractoriness was visually observed. In addition, simulation results were saved in the files for constructing time diagrams of the change in the transmembrane potential.

All the materials and experimental data in the article are presented in the online resource ResearchGate [11].

\section{RESULTS}

\subsection{Changing the Action Potential Waveform Depending on the Excitation Frequency}

Figure 1 shows the action potential waveform in a steady state at different frequencies of excitation impulses.

The action potential waveform was recorded at a hundredth impulse. The model parameters were initiated by finite values obtained for lower frequency of excitation impulses. It was possible to force an excitation rhythm of $240 \mathrm{~min}^{-1}$ on the model by increasing the excitation impulse frequency in the following sequence $60-180-220-230-240 \mathrm{~min}^{-1}$. It was impossible to force an excitation rhythm of more than 240 $\min ^{-1}$ on the models. Table 1 presents the success in forcing a high-frequency rhythm of excitation depending on the source and forced excitation impulse frequency.

The action potential waveform at an excitation impulse frequency of $60 \mathrm{~min}^{-1}$ is slightly different from that in a cardiomyocyte in a continuous resting state. As the frequency increases the duration of the action potential decreases, so does the duration of the resting state in relation to the action potential duration. If at a frequency of $60 \mathrm{~min}^{-1}$ the resting state duration takes up most of the impulse repetition period, then at a frequency of $240 \mathrm{~min}^{-1}$ most of the period is taken up by the action potential.

Examples of the results of forced rhythms are presented in Figure 2. The arrows in the figures show the action time of excitation impulses, the dashed line shows changes in the transmembrane potential after a rhythm was successfully forced.

In Figure 2(c), every second excitation impulse hits the refractoriness, and therefore no excitation of the cardiomyocyte model occurs. In fact, the model is excited at a frequency that is 2 times lower than the frequency of the excitation impulses.

The aim of a defibrillating pulse is to hinder fibrillation wave propagation in the myocardium. Fibrillation results in a high frequency of cardiomyocyte excitation in which, as is shown in Figure 1, the duration of the refractoriness is reduced. If the defibrillating pulse manages to prolong the refractoriness in 


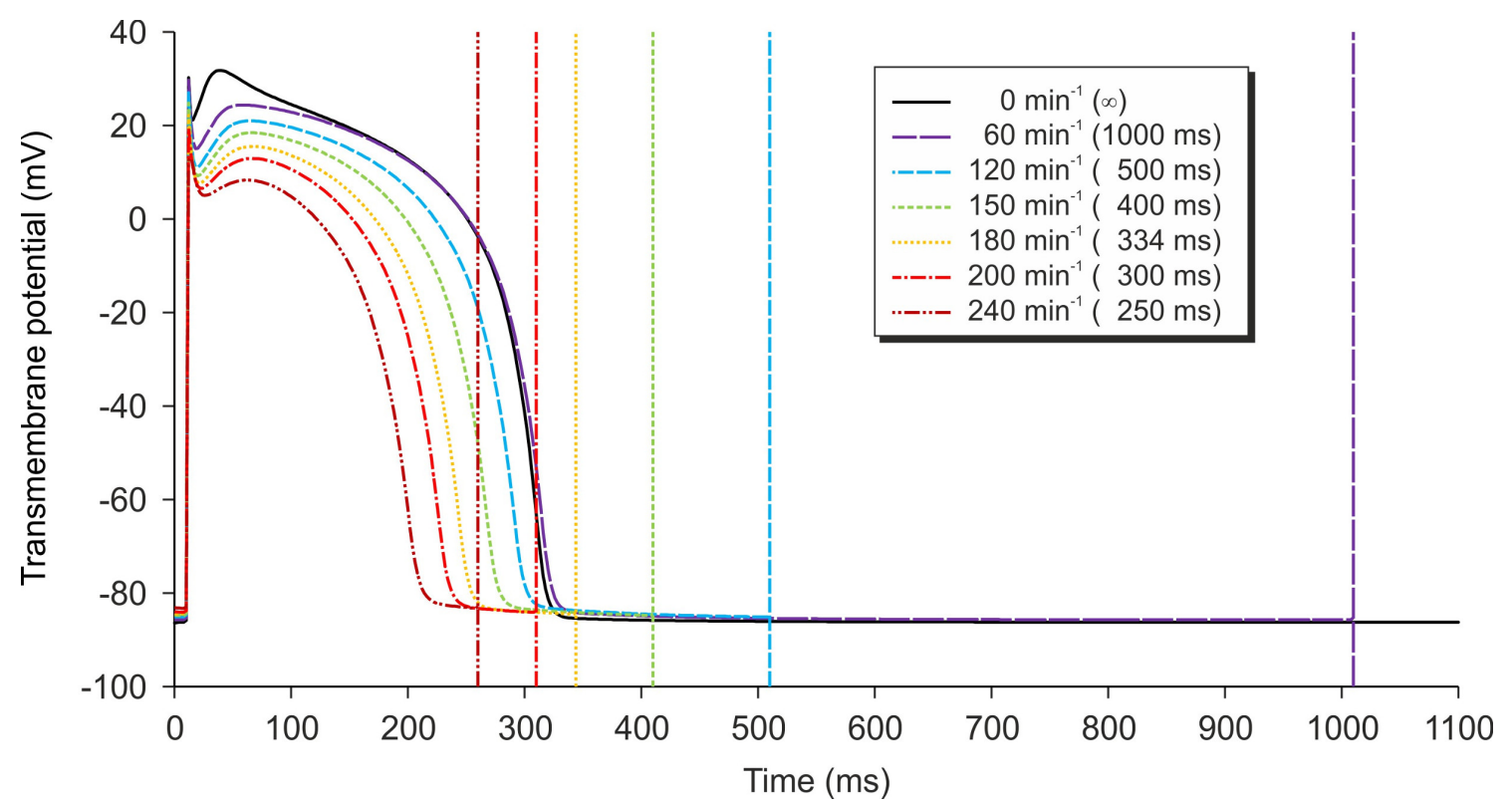

Figure 1. The action potential waveform at different frequencies (repetition periods) of excitation impulses. The vertical lines show the action time of the next excitation impulse.

Table 1. Success in forced excitation rhythm.

\begin{tabular}{|c|c|c|c|c|c|c|c|c|}
\hline \multirow{2}{*}{ Initial excitation impulse frequency, $\mathrm{min}^{-1}$} & \multicolumn{8}{|c|}{ Forced excitation impulse frequency, $\min ^{-1}$} \\
\hline & 120 & 150 & 180 & 200 & 210 & 220 & 230 & 240 \\
\hline 60 & yes & yes & yes & no & no & no & no & no \\
\hline 120 & & yes & yes & no & no & no & no & no \\
\hline 150 & & & yes & yes & yes & no & no & no \\
\hline 180 & & & & yes & yes & yes & * & no \\
\hline 200 & & & & & yes & yes & * & * \\
\hline 210 & & & & & & yes & * & * \\
\hline 220 & & & & & & & yes & $\star$ \\
\hline 230 & & & & & & & & yes \\
\hline
\end{tabular}

${ }^{\star}$ Greatly varying forms of related action potentials (alternation).

cardiomyocytes, it will block fibrillation wave propagation across the myocardium, and this wave will be destroyed. An assumption was made in [12] about the role of prolonging the refractoriness at the expense of recovering the cardiomyocyte excitation channel by a defibrillating pulse. The effect of prolonging the refractoriness was also obtained in experiments [13].

\subsection{Changing the Threshold Energy Ratio and the Duration of the Energetically Optimal Cardiomyocyte Excitation Impulse According to the Excitation Impulse Frequency}

The parameters for energy-optimal rectangular excitation impulses were determined for a cardiomyocyte both in the continuous resting state $\left(0 \mathrm{~min}^{-1}\right)$ and at excitation rhythms of 200 and $240 \mathrm{~min}^{-1}$. Figure 3 presents graphs showing the dependence of threshold energy ratios on excitation impulse 


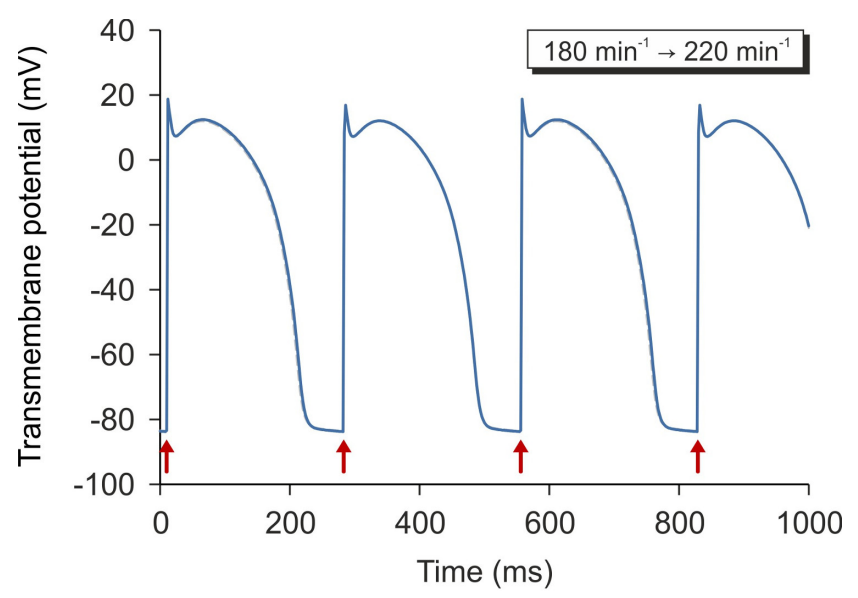

(a)

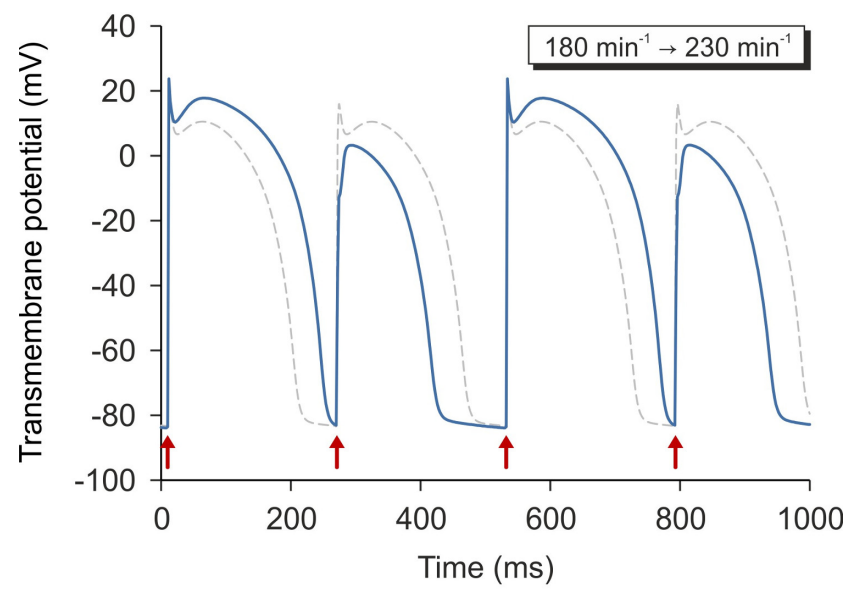

(b)

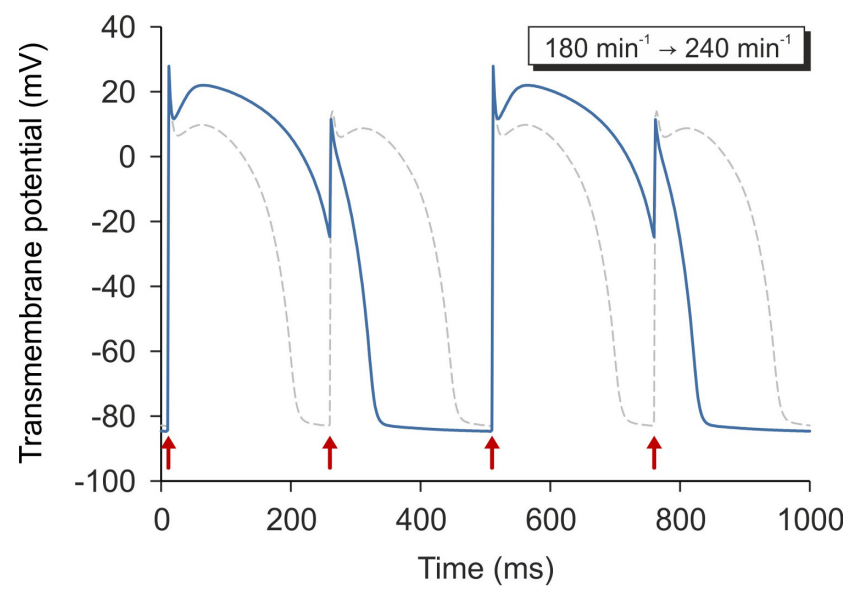

(c)

Figure 2. Examples of the results of forced rhythms. (a) A successfully forced rhythm when switching the excitation impulse frequency from 180 to $220 \mathrm{~min}^{-1}$; (b) Significantly different forms of the related action potential when switching the excitation impulse frequency from 180 to $230 \mathrm{~min}^{-1}$ (alternation); (c) The failure to force an excitation rhythm when switching the excitation impulse frequency from 180 to $240 \mathrm{~min}^{-1}$. 


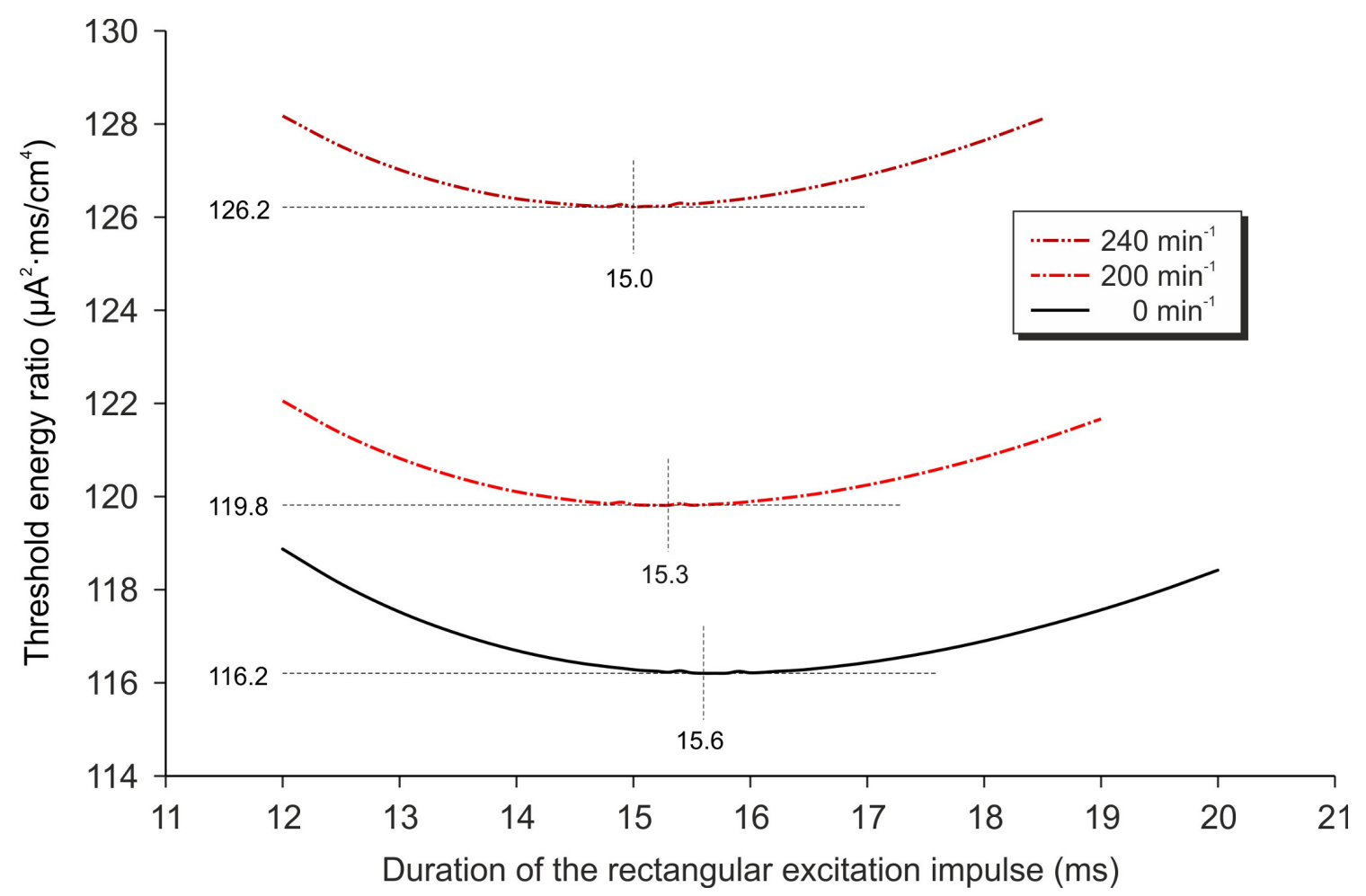

Figure 3. Dependence of the threshold excitation energy ratio on the rectangular impulse duration for the cardiomyocyte model in continuous resting state $\left(0 \mathrm{~min}^{-1}\right)$ and at excitation rhythms of 200 and $240 \mathrm{~min}^{-1}$.

duration at optimal values for these cases. The parameters of the energy-optimal excitation impulse at a rhythm of $240 \mathrm{~min}^{-1}$ differ insignificantly from those in a continuous resting state: optimal impulse duration is reduced by $5 \%$ and the threshold energy value is increased by $9 \%$.

\subsection{Threshold Impact}

Proceeding from the assumption that a defibrillating pulse prolongs refractoriness of cardiomyocytes, dependences of threshold values of the energy ratios of depolarizing rectangular pulses prolonging the refractoriness of the cardiomyocyte model, were built, depending on the delay relative to excitation impulse when defibrillating pulse duration was 15,30 and $45 \mathrm{~ms}$ at an excitation impulse frequency of $240 \mathrm{~min}^{-1}$ (Figure 4(a)).

Depending on the delay relative to the excitation impulse the depolarizing pulses had a different impact on the cardiomyocyte model. Four types of impacts were identified (Table 2). Table 3 gives ranges of delay values of the depolarizing pulse relative to the excitation impulse for different types of impact.

The parameters of the maximum and minimum values of threshold impacts are presented in Table 4.

Examples of the types of impact of depolarization pulse with $15 \mathrm{~ms}$ duration at an excitation impulse frequency of $240 \mathrm{~min}^{-1}$ are presented in Figure 5. The arrows in the figure show the action time of excitation impulses; the dashed line shows transmembrane potential changes at an excitation rhythm of 240 $\min ^{-1}$.

Figure 6 shows the diagrams of the change in the threshold energy ratio as a function of the duration of the depolarization pulse at delays in relation to the excitation impulse $70 \mathrm{~ms}$ (impact type B) and $160 \mathrm{~ms}$ (impact type D).

When the delay relative to the excitation impulse was $70 \mathrm{~ms}$ (impact type $\mathrm{B}$ ) the minimum threshold energy ratio was obtained when depolarizing pulse duration was $31 \mathrm{~ms}$, when the delay was $160 \mathrm{~ms}$ 


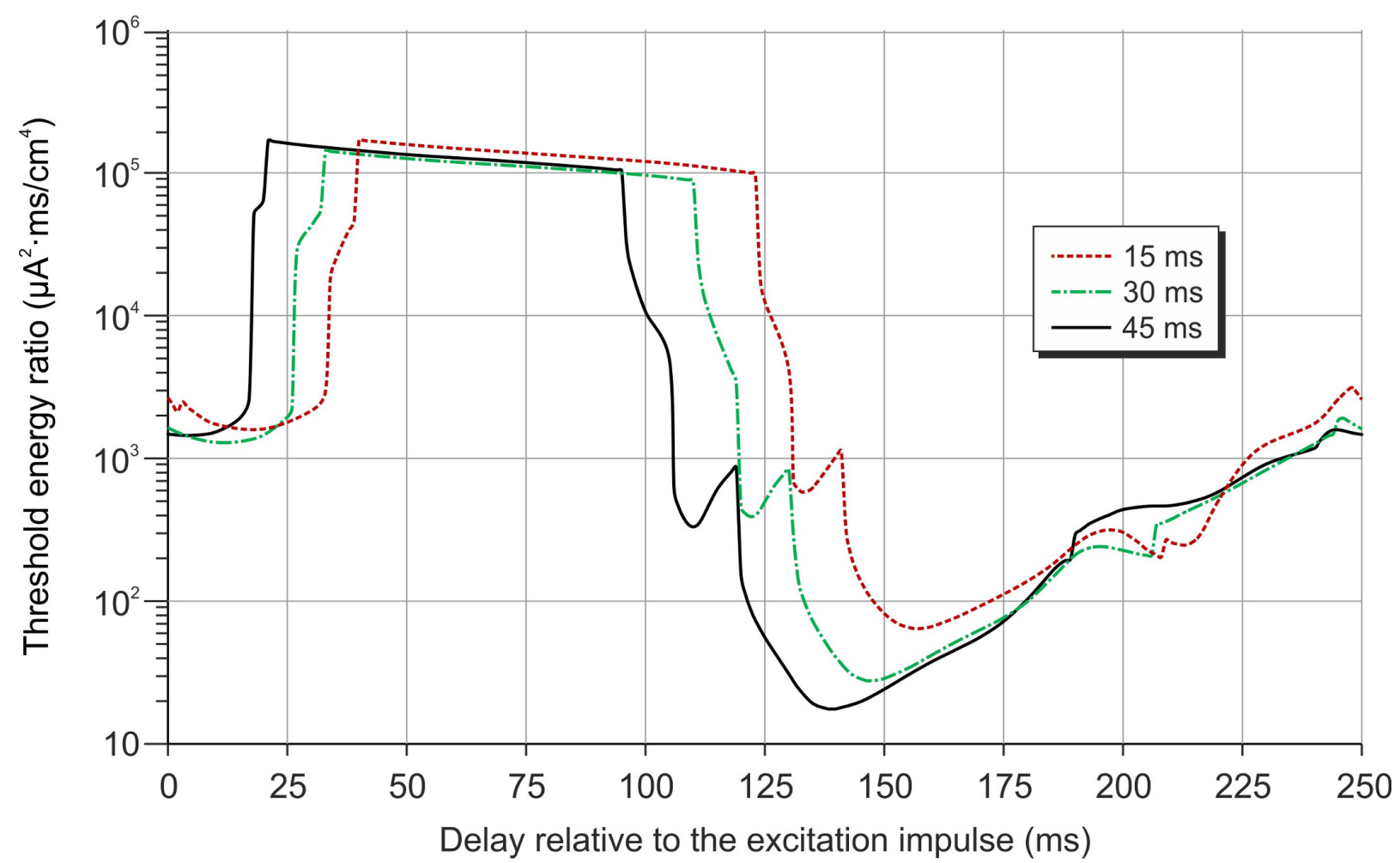

(a)

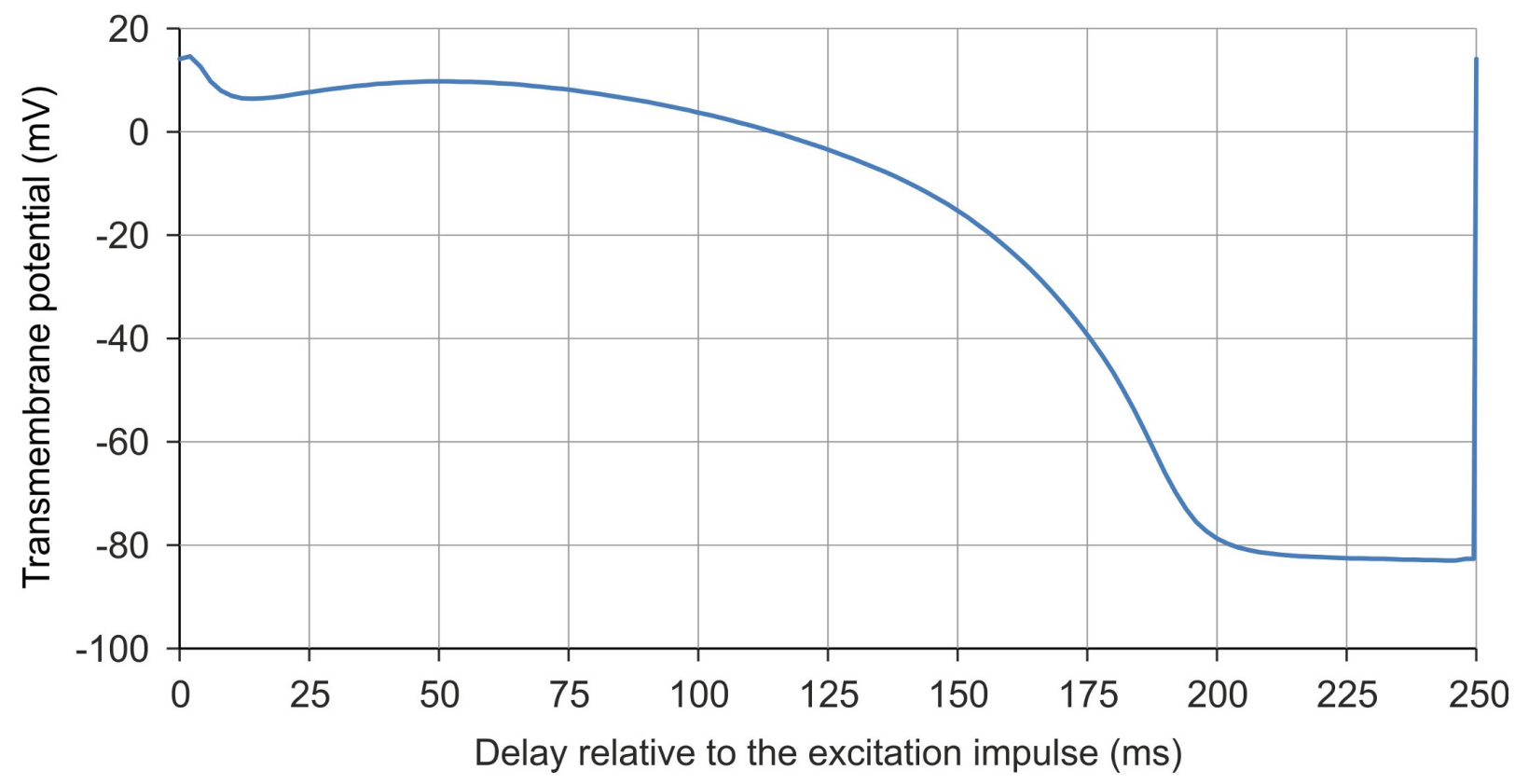

(b)

Figure 4. Dependences of threshold values of the energy ratios of depolarizing rectangular pulses prolonging the refractoriness of the cardiomyocyte model depending on the delay relative to excitation impulse when pulse duration is 15,30 and $45 \mathrm{~ms}$ at an excitation impulse frequency of 240 $\mathrm{min}^{-1}$; (b) The cycle of changes in the transmembrane potential of the cardiomyocyte model at excitation impulse frequency of $240 \mathrm{~min}^{-1}$. 
Table 2. Types of depolarizing pulse impact on the cardiomyocyte model.

Designation of impact type

A

B

C

$\mathrm{D}$
Impact description

Shortening of the current refractoriness causes the next one to be longer

High-energy suppression of the next refractoriness causes a longer third refractoriness

Shortening of the next refractoriness causes a longer third refractoriness

Low-energy suppression of the next refractoriness causes a longer third refractoriness

Table 3. Types of depolarizing pulse impact on the cardiomyocyte model.

\begin{tabular}{ccccc}
\hline \multirow{2}{*}{$\begin{array}{c}\text { Depolarizing pulse } \\
\text { duration, ms }\end{array}$} & \multicolumn{4}{c}{$\begin{array}{c}\text { Value ranges of delay of the depolarizing pulse relative } \\
\text { to the excitation impulse, ms, for different types of impacts }\end{array}$} \\
\cline { 2 - 5 } & A & B & C & D \\
\hline 15 & $0-39$ & $40-123$ & $124-141,209-250$ & $142-208$ \\
30 & $0-32$ & $33-110$ & $111-130,207-250$ & $131-206$ \\
45 & $0-20$ & $21-95$ & $96-119,190-250$ & $120-189$ \\
\hline
\end{tabular}

Table 4. Parameters of maximum and minimum values of threshold impacts.

Parameter

\begin{tabular}{|c|c|c|c|}
\hline \multirow{2}{*}{ Parameter } & \\
\hline & 15 & 30 & 45 \\
\hline & \multicolumn{3}{|c|}{ Maximum threshold value } \\
\hline Delay relative to excitation impulse, $\mathrm{ms}$ & 40 & 33 & 21 \\
\hline Current amplitude, $\mu \mathrm{A} / \mathrm{cm}^{2}$ & 106 & 69 & 61 \\
\hline \multirow[t]{2}{*}{ Energy ratio, $\mu \mathrm{A}^{2} \cdot \mathrm{ms} / \mathrm{cm}^{4}$} & 170000 & 143000 & 168000 \\
\hline & \multicolumn{3}{|c|}{ Minimal threshold value } \\
\hline Delay relative to excitation impulse, $\mathrm{ms}$ & 157 & 147 & 139 \\
\hline Current amplitude, $\mu \mathrm{A} / \mathrm{cm}^{2}$ & 2.07 & 0.96 & 0.63 \\
\hline Energy ratio, $\mu \mathrm{A}^{2} \cdot \mathrm{ms} / \mathrm{cm}^{4}$ & 64 & 28 & 18 \\
\hline
\end{tabular}



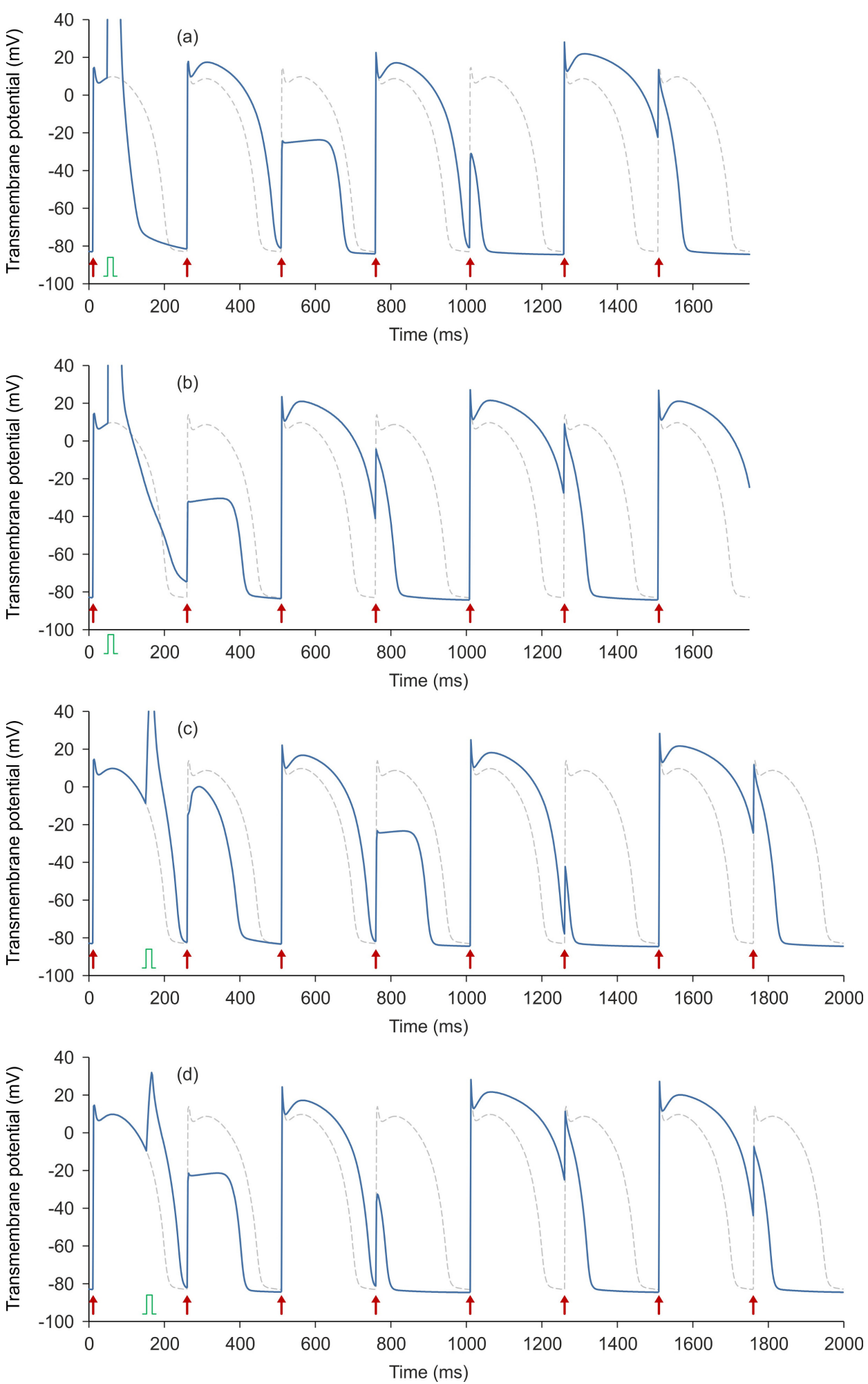

Figure 5. Examples of the types of impact of depolarization pulse with a duration of 15 $\mathrm{ms}$ at a excitation impulse frequency of $240 \mathrm{~min}^{-1}$. (a) Impact type $\mathrm{A}$, the delay relative to the excitation impulse is $39 \mathrm{~ms}$; (b) Impact type $B$, the delay relative to the excitation impulse is $40 \mathrm{~ms}$; (c) Impact type $C$, the delay relative to the excitation impulse is 141 ms; (d) Impact type $D$, the delay relative to the excitation impulse is $142 \mathrm{~ms}$. 


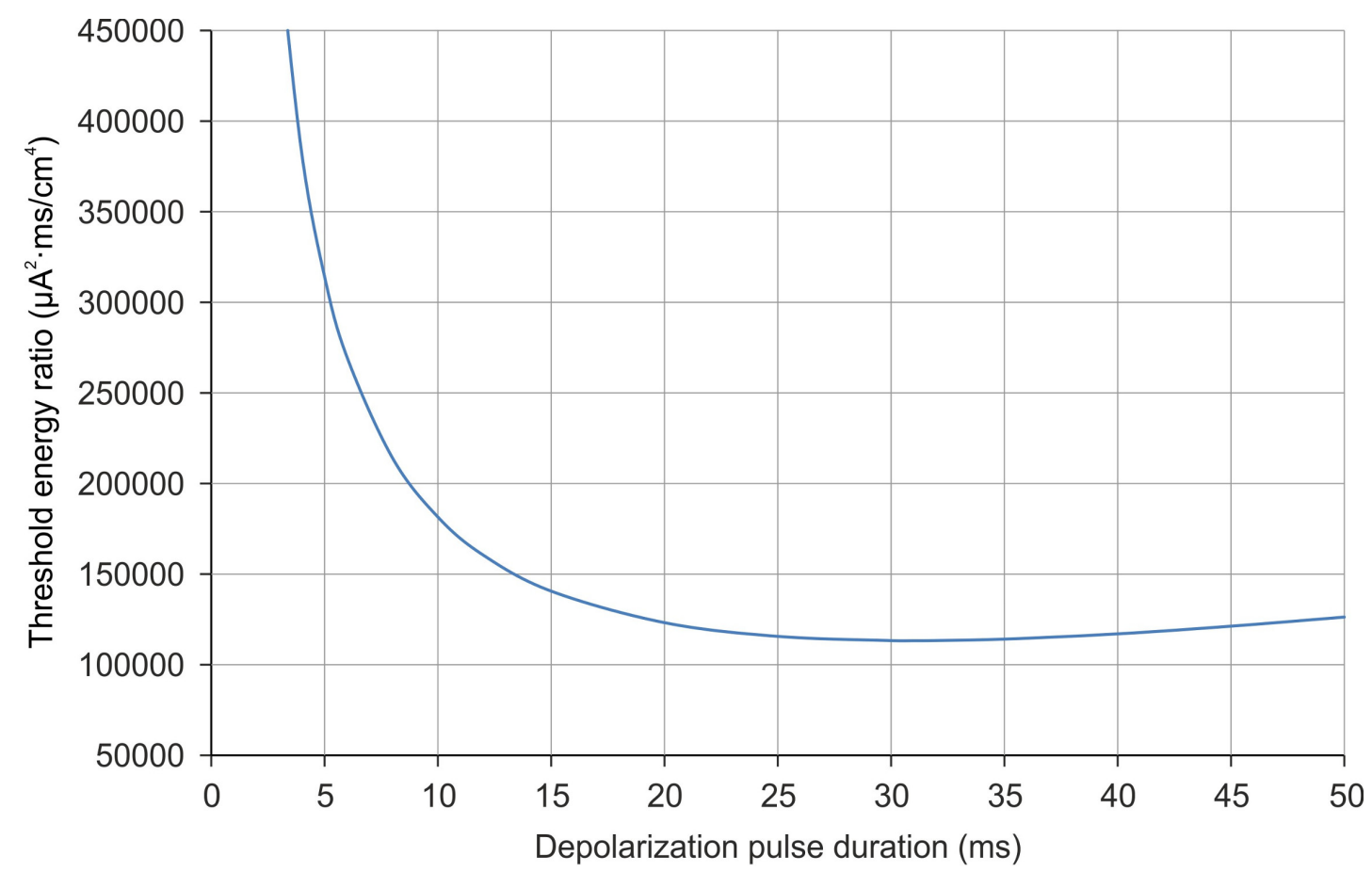

(a)

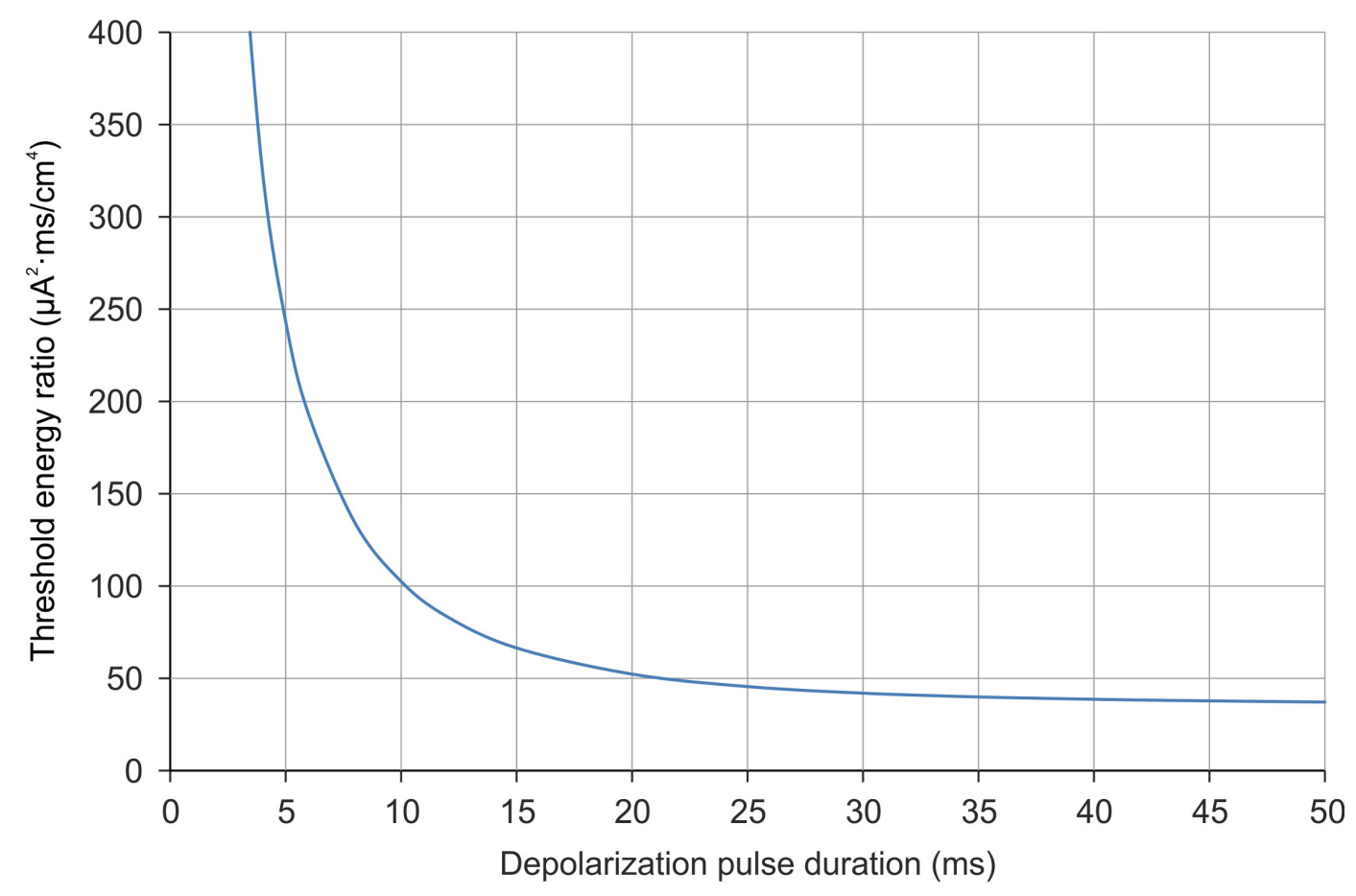

(b)

Figure 6. Examples of the types of impact of depolarization pulse with a duration of 15 $\mathrm{ms}$ at a excitation impulse frequency of $240 \mathrm{~min}^{-1}$. (a) Impact type $\mathrm{A}$, the delay relative to the excitation impulse is $39 \mathrm{~ms}$; (b) Impact type $B$, the delay relative to the excitation impulse is $40 \mathrm{~ms}$; (c) Impact type $C$, the delay relative to the excitation impulse is 141 ms; (d) Impact type $D$, the delay relative to the excitation impulse is $142 \mathrm{~ms}$. 
(impact type D) the threshold energy ratio decreased as the pulse duration increased in the entire range of the values studied.

\section{DISCUSSION}

The study found only one mechanism that explains the impact of defibrillating pulses on the cardiomyocyte-that of prolonging its refractoriness.

The study revealed differences in the mechanisms of action on cardiomyocytes between excitation and defibrillation impulses. If the threshold energy ratio of the excitation impulse is $\sim 120 \mu \mathrm{A}^{2} \cdot \mathrm{ms} / \mathrm{cm}^{4}$ at the optimal duration of $\sim 15 \mathrm{~ms}$, then threshold energy ratio is $\sim 140,000 \mu \mathrm{A}^{2} \cdot \mathrm{ms} / \mathrm{cm}^{4}$ in the phase of the high-energy defibrillation at an optimal pulse duration of $\sim 30 \mathrm{~ms}$. In the phase of low-energy defibrillation the threshold energy ratio decreases as pulse duration increases and is $\sim 20 \mathrm{MK} \mathrm{A}^{2} \cdot \mathrm{Mc} / \mathrm{cm}^{4}$ at a pulse duration of $45 \mathrm{~ms}$.

The duration of energetically optimal excitation impulse $(15 \mathrm{~ms})$ is higher than that obtained for the Luo-Rudy model (11 ms) [14].

Impact types A and B are presumably associated with classical defibrillation in which the energy of a monophasic pulse can be as high as $360 \mathrm{~J}$. However, the duration value of an energetically optimal defibrillating pulse $(\sim 30 \mathrm{~ms})$ on the model significantly differs from the energetically optimal duration of the human defibrillating pulse obtained experimentally (approx. $4 \mathrm{~ms}$ [15]). Impact types $\mathrm{C}$ and $\mathrm{D}$ explain the efficacy of low-energy defibrillation [16].

Low-energy defibrillation is extremely attractive for use in clinical practice. With external cardioversion, it can solve the problem of exceeding the pain threshold, since current of the pulse of cardioversion becomes many times smaller and lower than the pain threshold. As a result, the use of medication for pain relief before cardioversion will not be required. Also, the reducing of energy necessary for defibrillation arrives to reduce the size and weight of the power module of the defibrillator and its cost has been reduced. However, the possibility of low-energy defibrillation requires further theoretical studies and experiments on animals.

\section{CONCLUSIONS}

The cardiomyocyte model has a limited susceptibility to a forced rhythm of high frequency excitation. It is possible to force a high frequency excitation rate by increasing the excitation impulses frequency gradually.

Presumably, the mechanism of defibrillation pulse impact is the prolongation of the refractoriness of cardiomyocytes which undermines their susceptibility to a forced high-frequency fibrillation rhythm for a long time, as a result for which they hinder the propagation of a fibrillation wave. This is the only defibrillation mechanism that has been identified during the simulation.

The threshold energy of a defibrillation depolarizing pulse that causes a longer cardiomyocyte refractoriness varies depending on a delay relative to the excitation impulse (the excitation phase) on a wide range, with the maximum value exceeding the minimum one as much as several thousand times).

The results show that the excitation impulse and the monophasic defibrillation pulse have different mechanisms of impact on cardiomyocyte.

Myocardial cardiomyocytes are in different phases of the excitation cycle in the process of fibrillation wave propagation. The study suggests that applying a series of low-energy defibrillating pulses during a repetition period of excitation impulses will violate susceptibility of the myocardium myocytes to the forced high-frequency fibrillation rhythm in amounts large enough to destroy the fibrillation wave. The effectiveness of this defibrillation method has been confirmed by experimental studies [16].

\section{ACKNOWLEDGEMENTS}

The author is grateful to I. V. Biktasheva (University of Liverpool, UK) and V. N. Biktashev (Univer- 
sity of Exeter, UK) for their advice on the use of the BeatBox simulation environment. This work was funded by Ministry of Education and Science of the Russian Federation according to the government research project № 12.2418.2014/K.

\section{CONFLICTS OF INTEREST}

The author declares that there is no conflict of interest regarding the publication of this article.

\section{REFERENCES}

1. Kroll, M.W. (1993) A Minimal Model of the Monophasic Defibrillation Pulse. Pacing and Clinical Electrophysiology, 16, 769-777. https://doi.org/10.1111/j.1540-8159.1993.tb01657.x

2. Walcott, J.P., Walker, R.G., Cates, A.W., Krassowska, W., Smith, W.M. and Ideker, R.E. (1995) Choosing the Optimal Monophasic and Biphasic Waveforms for Ventricular Defibrillation. Journal of Cardiovascular Electrophysiology, 6, 737-750. https://doi.org/10.1111/j.1540-8167.1995.tb00450.x

3. Fishler, M.G. (2000) Theoretical Predictions of the Optimal Monophasic and Biphasic Defibrillation Waveshapes. IEEE Transactions on Biomedical Engineering, 47, 59-67. https://doi.org/10.1109/10.817620

4. Krasteva, V., Cansell, A. and Daskalov, I. (2000) Modelling Transthoracic Defibrillation Waveforms. Journal of Medical Engineering \& Technology, 24, 63-67. https://doi.org/10.1080/030919000409320

5. Gorbunov, B.B. (2009) Characteristic Energy Method for Evaluation of Monopolar Defibrillation Pulse Efficiency. Biomedical Engineering, 43, 56-61. https://doi.org/10.1007/s10527-009-9097-5

6. Vostrikov, V.A., Gorbunov, B.B., Gusev, A.N., Nesterenko, I.V. and Selishchev S.V. (2013) Determination of Threshold Energy Level of Monopolar Defibrillation Pulses Using the Luo-Rudy Cardiomyocyte Model. Biomedical Engineering, 47, 61-64. https://doi.org/10.1007/s10527-013-9335-8

7. Vostrikov, V.A., Gorbunov, B.B., Gusev, A.N. (2014) Computer Simulation of Cardiomyocyte Membrane Exposure to First-Phase Bipolar Defibrillation Impulses. General Reanimatology, 10, 29-32. https://doi.org/10.15360/1813-9779-2014-1-25-32

8. Vostrikov, V.A., Gorbunov, B.B. and Selishchev, S.V. (2015) Construction of Energy-Optimal Smooth Monophasic Defibrillation Pulse Waveforms Using Cardiomyocyte Membrane Model. Journal of Biomedical Science and Engineering, 8, 625-631. https://doi.org/10.4236/jbise.2015.89058

9. Antonioletti, M., Biktashev, V.N., Jackson, A., Kharche, S.R., Stary, T. and Biktasheva, I.V. (2017) BeatBox HPC Simulation Environment for Biophysically and Anatomically Realistic Cardiac Electrophysiology. PLoS One, 12, e0172292. https://doi.org/10.1371/journal.pone.0172292

10. ten Tusscher, K.H.W.J. and Panfilov, A.V. (2006) Alternans and Spiral Breakup in a Human Ventricular Tissue Model. American Journal of Physiology_Heart and Circulator, 291, H1088-H1100.

https://doi.org/10.1152/ajpheart.00109.2006

11. Gorbunov, B.B. (2017) Study the Impact of the Current Rectangular Pulses at a ten Tusscher-Panfilov Model of Human Ventricular Myocyte.

https://www.researchgate.net/project/Study-the-Impact-of-the-Current-Rectangular-Pulses-at-a-ten-Tusscher-P anfilov-Model-of-Human-Ventricular-Myocyte

12. Swartz, J.F., Jones, J.L., Jones, R.E. and Fletcher, R. (1991) Conditioning Prepulse of Biphasic Defibrillator Waveforms Enhances Refractoriness to Fibrillation Wavefronts. Circulation Research, 68, 438-449. https://doi.org/10.1161/01.RES.68.2.438

13. Li, H.G., Jones, D.L., Yee, R. and Klein, G.J. (1995) High Voltage Shock Induced Cellular Electrophysiological Effects: Transient Refractoriness and Bimodal Changes in Action Potential Duration. Pacing and Clinical Elec- 
trophysiology, 18, 1225-1235. https://doi.org/10.1111/j.1540-8159.1995.tb06962.x

14. Gorbunov, B.B. (2012) A Study of the Myocardium Cell Membrane Using the Luo-Rudy Model. Biomedical Engineering, 46, 117-119. https://doi.org/10.1007/s10527-012-9282-9

15. Cansell, A. (1998) Wirksamkeit und Sicherheit der Impulskurvenformen bei transthorakaler Defibrillation. Notfall \& Rettungsmedizin, 1, 372-380. https://doi.org/10.1007/s100490050087

16. Janardhan, A.H., Li, W., Fedorov, V.V., Yeung, M., Wallendorf, M.J., Schuessler, R.B. and Efimov, I.R. (2012) A novel Low-Energy Electrotherapy That Terminates Ventricular Tachycardia with Lower Energy than a Biphasic Shock When Antitachycardia Pacing Fails. Journal of the American College of Cardiology, 60, 2393-2398. https://doi.org/10.1016/j.jacc.2012.08.1001

Submit or recommend next manuscript to SCIRP and we will provide best service for you:

Accepting pre-submission inquiries through Email, Facebook, LinkedIn, Twitter, etc.

A wide selection of journals (inclusive of 9 subjects, more than 200 journals)

Providing 24-hour high-quality service

User-friendly online submission system

Fair and swift peer-review system

Efficient typesetting and proofreading procedure

Display of the result of downloads and visits, as well as the number of cited articles

Maximum dissemination of your research work

Submit your manuscript at: http://papersubmission.scirp.org/

Or contact jbise@scirp.org 